\title{
Missed Lipoma of the Spermatic Cord
}

\section{Yener O., Demir M., Yigitbașı R., Yilmaz A.}

Department of Surgery, Göztepe Training and Research Hospital, Istanbul, Turkey

Received March 28, 2012; Accepted January 15, 2013.

Key words: Inguinal hernia - Spermatic cord lipoma

Abstract: The aim of the research was to determine the incidence, significance, and anatomy of spermatic cord and round ligament lipomas. Between 2000 and 2010 we evaluated 969 consecutive patients with 1,070 indirect inguinal hernias, who underwent open repair. A total of 22 lipomas of the spermatic cord or round ligament were identified and resected in 22 patients. No neoplastic changes confirmed in histopathologic examinations of the specimens were reported. Lipomas of the cord and round ligament occur with a considerable incidence. We believe that even if there is no peritoneal sac, the herniation of extraperitoneal fat through the inguinal canal should be counted as an inguinal hernia, and it requires adequate treatment.

Mailing Address: Dr. Oktay Yener, Department of Surgery, Göztepe Training and Research Hospital, Plaj yolu yıldız sok. Kaya apt. No:16 D:10 caddebostan, Istanbul, Turkey; Mobile Phone: 905337088 469; e-mail: oktayener@gmail.com 


\section{Introduction}

Fatty tissue within the internal cremasteric fascia is frequently encountered during hernia surgery, and it is called a cord lipoma in the surgical literature. Lipoma of the cord is the most common benign tumour of the spermatic cord and a common condition of the male population (Lilly and Arregui, 2002).

It is frequently under-diagnosed and ignored because of its benign course. Clinical series have reported an incidence of $22.5 \%$ during inguinal hernia surgery (Fawcett and Rooney, 1997). Cord lipoma, fatty tissue lying in the internal cremasteric fascia, is frequently encountered during both open and laparoscopic inguinal hernia surgery. Although cord lipoma is familiar to surgeons, not much research has been published about it until recently. Incidence and significance of cord lipoma in hernia patients or in the nonherniated population has been stressed in the past few years.

\section{Methods}

Between 2000 and 2010, 969 consecutive patients with indirect inguinal hernias underwent open repair. Recurrent hernias were not included. Data about age, sex, hernia type, and presence of an inguinal cord lipoma were recorded. We defined cord lipoma as fatty tissue lying in the inguinal canal, which is separable from the surrounding cord structures and distinct from the fatty tissue accompanying the testicular vessels. Hernia types were determined during surgery by using the classification described by Nyhus et al. (1991). All lipomas were resected with or without the peritoneal sac, and the fatty materials were sent to the laboratory for histologic examination. The statistical analyses used were the Student's $t$-test for ordinal data.

\section{Results}

A total of 969 hernia repairs were performed, 747 on male patients and 222 on female patients, with an average age of 52.8 years (range 17-70). A total of 22 lipomas of the spermatic cord or round ligament were identified. Hernias were classified as indirect, direct, pantaloon (combined direct and indirect), femoral, or none. There were 768 indirect, 152 direct, 47 pantaloon hernia and 22 lipomas. The overall incidence of cord lipoma in those explored for hernia repair was $3.4 \%$.

Twenty-two cord lipomas were identified in patients who did not have a hernia defect. Of these lipomas, 19 were in male patients and 3 in female patients. Fourteen of these were symptomatic and eight had no symptoms of pain but did have a palpable bulge.

Lipomas were noted to protrude through the internal inguinal ring to lie on the anterolateral aspect of the spermatic cord, much like an indirect hernia. When viewed from a preperitoneal dissection, a filmy connection containing the blood supply could be visualized to extend to the retroperitoneum, inferior to the cord 
structures. The lipoma and blood supply was found outside the internal spermatic fascia. Histopathologic examination of all the specimens was reported as mature adipose tissue, indicating that the cord lipomas were not neoplastic in origin.

\section{Discussion}

The use of the term lipoma for fatty tissue of the spermatic cord or round ligament causes some confusion in the surgical literature. Although fat within the cord is often recorded as a lipoma, even in pathology reports, there usually are no neoplastic or degenerative changes noted in the resected fat. A cord lipoma is merely normal fat, as confirmed in this study and in that of Heller and colleagues (2002). Another source of confusion arising from the presence of a "lipoma" is the question of whether this entity is just an anatomic variation or a true hernia (Rosenberg, 1979).

While most authors agree that a cord lipoma is a protrusion of extraperitoneal fatty tissue through the internal inguinal ring, it is described as herniated extraperitoneal fat but not as a true hernia (McDonnell and Walsh, 2000). In terms of the aetiology of hernia, the presence of a peritoneal protrusion in the inguinal canal does not always define a clinical hernia. Sometimes a peritoneal sac is not evident (Read and Schaefer, 2000). In other situations, a fine peritoneal tube can be present, as in a patent processus vaginalis and canal of Nuck, without any bowel or other content. If we consider people with a patent processus vaginalis as not necessarily having an inguinal hernia, then we can say that a peritoneal protrusion is not the only determining factor in development of an inguinal hernia. As stated by Condon, the causes of indirect inguinal hernia are structural variations of transversalis fascia and the transversus abdominis aponeurosis, which determine the strength and size of the deep inguinal ring (Bailey and Love, 1997). The sphincter mechanism of the internal ring cannot resist the elevated intra-abdominal pressure. Some tissues, located in the musculoaponeurotic borders of the abdomen, overflow through the inguinal canal, which is a parietal peritoneum and/or extraperitoneal fat tissue. Probably one is the leading factor for the other between them. The amount of fat in the extraperitoneal space is variable and reflects the fat content of the body.

Rosenberg stated that in middle-aged patients, indirect inguinal hernias are almost universally associated with a lipoma or other extraperitoneal fat adherent to the sac (Condon, 1995). While the saccular theory of Russell is still important in the history of hernia (Russell, 1906), the content within the inguinal canal determining the presence or absence of an indirect inguinal hernia should not be limited to bowel or omentum. Cord lipomas are in continuity with the extraperitoneal fat, and this continuity can be clearly seen during laparoscopic repair. Protrusion through the internal inguinal ring of extraperitoneal fat or of peritoneum with its content are both capable of producing the symptoms and signs of an inguinal hernia and should be considered equally as important as consequences of distorted 
regional anatomy. That is, both herniated extraperitoneal fat and herniated peritoneum and its contents are true inguinal hernias.

By the nature of our definition of cord lipomas, they are all "indirect". The incidence of cord lipoma was greater among patients with indirect hernia defects as opposed to those with direct defects.

Although it is difficult to say that this represents a cause-and-effect relationship, it is possible that lobular retroperitoneal fat (i.e. lipoma) insinuates itself through the internal ring and over time dilates it. This could then predispose to indirect hernia formation. In our series we showed that in many instances the lipoma is the only pathology identified during an exploration for a hernia repair. It is quite clear in these situations that the lipoma needs to be resected. The question is what can be extrapolated from those situations to the incidental lipomas that are found at the time of hernia repair when a true hernia is also identified. Lipomas of the spermatic cord or round ligament have been shown to be a true pathology of the groin, because they can be the etiology of significant symptoms. A lipoma of the cord is herniated preperitoneal fat, which causes symptoms as a true hernia, which is herniated peritoneal content. Whether found with or without a hernia at exploration for hernia repair, they must be dealt with. This does not imply that lipomas that are asymptomatic and found incidentally by ultrasound or intraoperative examination during contralateral repair should be resected, but they should certainly be annotated in case of future symptoms.

We believe that even if there is no peritoneal sac, the herniation of extraperitoneal fat through the inguinal canal should be counted as an inguinal hernia, and it requires treatment.

\section{References}

Bailey, H., Love, M. (1997) Bailey and Love's Short Practice of Surgery, 22 ${ }^{\text {nd }}$ Ed. (Chap. 55, p. 887: Hernias, umbilicus, abdominal wall) Chapman and Hall Medical, London.

Condon, R. E. (1995) The anatomy of the inguinal region and its relation to groin hernia. In: Hernia, $4^{\text {th }}$ Ed.

Nyhus, L. M., Condon, R. E., Pp. 16-72, JB Lippincott Co., Philadelphia.

Fawcett, A. N., Rooney, P. S. (1997) Inguinal cord lipoma. Br.J. Surg. 84, 1169.

Heller, C. A., Marucci, D. D., Dunn, T., Barr, E. M., Houang, M., Dos Remedios, C. (2002) Inguinal canal “lipoma”.

Clin. Anat. 15, 280-285.

Lilly, M. C., Arregui, M. E. (2002) Lipoma of the cord and round ligament. Ann. Surg. 235, 586-590.

McDonnell, C. O., Walsh, T. N. (2000) A hernia sac cannot be found at operation. Br. J. Surg. 87, 521-522.

Nyhus, L. M., Bombeck, C. T., Klein, M. S. (1991) Hernias. In: Textbook of Surgery: The Biological Basis of Modern

Surgical Practice, 14th Ed. Sabiston, D. C., Pp. 1134-1148, WB Saunders, Philadelphia.

Read, R. C., Schaefer, R. F. (2000) Lipoma of the spermatic cord, fatty herniation, liposarcoma. Hernia 4, 149-154.

Rosenberg, N. (1979) “Lipoma” of the spermatic cord: Potential relationship to indirect inguinal hernia in adults. Arch. Surg. 114, 549-550.

Russell, R. H. (1906) The saccular theory of hernia and the radical operation. Lancet 3, 1197-1203. 